\title{
Skeletal Muscle Quality Beyond Average Muscle Attenuation: A Proposal of Skeletal Muscle Phenotypes to Predict Short-Term Survival in Patients With Endometrial Cancer
}

\author{
Camila Santos Rodrigues, MD, and Gabriela Villaça Chaves, $\mathrm{PhD}$
}

\begin{abstract}
Background: Increasing evidence links sarcopenia and cancer prognosis, but limited data have focused on whether and to what extent muscle radiodensity can impact cancer outcomes. This study was conducted to investigate whether skeletal muscle mass, when divided into subranges of low or high radiodensity, improves prediction of short-term survival in patients with endometrial cancer (EC). Four skeletal muscle phenotypes were proposed to assess which is the best predictor of 1-year mortality. Methods: Patients with EC who had CT images available within 30 days before treatment $(n=208)$ were enrolled in a retrospective cohort. CT images at the third lumbar vertebra ( $L 3)$ were used to assess overall skeletal muscle index (SMI), which was then divided into subranges of radiation attenuation: low- and high-radiodensity SMI. The average muscle radiation attenuation (AMA) was also assessed. SMI and AMA were categorized as below or above the median and as below or above 30 Hounsfield units $(\mathrm{HU})$, respectively, to construct 4 skeletal muscle phenotypes: "high SMI + high AMA"; "low SMI + high AMA"; "high SMI + low AMA"; and "low SMI + low AMA". One-year survival was evaluated using the Kaplan-Meier method and Cox multiple regression analysis. Results: All of the skeletal muscle parameters, except the SMI, were significantly associated with shorter 1-year survival. The skeletal muscle phenotype of "low SMI + low AMA" showed the strongest association with 1-year mortality (hazard ratio, 5.36; $95 \% \mathrm{Cl}, 1.70-16.51)$. Conclusions: The additional value of classifying the skeletal muscle into subranges of radiodensity should be explored in the future. Evaluating the impact of skeletal muscle phenotypes on cancer prognosis is promising and must be assessed in further studies.
\end{abstract}

Sarcopenia was first described as a geriatric syndrome with multifactorial etiology, consisting of low muscle mass combined with low muscle strength or impaired physical performance. ${ }^{1}$ A decrease in muscle mass and strength have also been described, however, in different situations related to lifestyle, chronic diseases, and cancer therapy. ${ }^{2}$

The impact of sarcopenia on unfavorable outcomes in patients with cancer, such as greater risks of chemotherapy toxicity ${ }^{3}$ and surgical complications, ${ }^{4}$ as well as shorter survival, ${ }^{5}$ has been widely demonstrated in

From the Brazilian National Cancer Institute, Rio de Janeiro, Brazil. Submitted May 4, 2017; accepted for publication August 29, 2017. The authors have disclosed that they have no financial interests, arrangements, affiliations, or commercial interests with the manufacturers of any products discussed in this article or their competitors. the literature on different types of tumors and cancer stages. More recently, myosteatosis, characterized by increased muscle fat content rather than low muscle mass, has been associated with shorter survival and/or surgical complications in patients with cancer. ${ }^{6-8}$ Although the gold standard method for diagnosing myosteatosis is muscular biopsy, diagnosis can also be made using imaging techniques (eg, CT), ${ }^{9}$ given that an inverse association between muscle radiation attenuation and triglyceride content in muscle phantoms and muscle biopsies has been described..$^{10}$

Author contributions: Study concept and design: Chaves. Data acquisition: Rodrigues. Data analysis and interpretation: Rodrigues. Manuscript preparation: Rodrigues, Chaves.

Correspondence: Gabriela Villaça Chaves, PhD, Brazilian National Cancer Institute, Rua Equador 831, Rio de Janeiro, RJ 20220-410, Brazil. E-mail: gabrielavc@gmail.com 
Rodrigues and Chaves

In the oncology setting, determination of body composition via CT has been considered the reference method, because this examination is routinely performed for diagnosis, staging, and follow-up. ${ }^{2}$ Identification and quantification of tissues through imaging methods consider the standard values for the radiodensity of each tissue, expressed in Hounsfield units (HU). Predefined radiation attenuation ranges are used to demarcate adipose tissue (from -190 to $-30 \mathrm{HU})$ and muscle $(-29$ to $+150 \mathrm{HU}) .{ }^{11}$

Although the HU range that characterizes adipose tissue is well established, ${ }^{12}$ there is disagreement in the literature as to which range would be adequate for characterization of skeletal muscle tissue. The generally accepted lower boundary of normal attenuation muscle is between +30 and +150 HU. ${ }^{6}$ Some authors do not consider the interval between -29 and $+29 \mathrm{HU}$ as a muscle attenuation range, whereas others disregard the range between -29 and +0 HU. ${ }^{9,10,13}$ However, using this approach, any region within this attenuation range is regarded as being neither muscle nor adipose tissue, which means that a significant proportion of muscle content appraised on CT images in certain individuals would be omitted. ${ }^{6}$ Although skeletal muscle tissue has been determined to be within the range of -29 to $+150 \mathrm{HU}$, it is suggested that the range between -29 and +29 HU should be used to identify skeletal muscle mass with low muscle radiation attenuation. ${ }^{6}$

Thus far, studies that have assessed the relationship of low muscle radiation attenuation in clinical and surgical results have compared HU mean values, assuming that higher average $\mathrm{HU}$ values indicate lower muscle adipose tissue infiltration and higher muscle strength. ${ }^{14-16}$ It has not been described whether the magnitude of the cross-sectional area of high-and low-radiodensity skeletal muscle is related to oncologic prognosis.

Hence, our aim was to investigate whether skeletal muscle mass, when divided into subranges of low- or high-radiodensity, improves the prediction of short-term survival in patients with endometrial cancer (EC) when compared with the average muscle attenuation and the standard skeletal muscle radiodensity used for sarcopenia diagnosis. Additionally, we proposed 4 skeletal muscle phenotypes to assess which one is the best predictor of 1-year mortality in this group.

\section{Methods}

This retrospective cohort study included all new patients referred to a cancer treatment institute in Brazil from October 1, 2008, to December 31, 2014, who had a confirmed diagnosis of EC and had CT scans taken up to 30 days before treatment. Women previously diagnosed with another type of cancer or those with synchronous tumors were excluded from the study, as were those whose lack of CT scans due to hardware artifact or image quality. This study was approved by the Ethics and Research Committee of the Brazilian National Cancer Institute (466.070/2013).

A total of 1,383 women with suspected EC were registered during the study period, of which 1,039 were excluded because they did not have CT scans performed before treatment. Case records of the remaining 344 women who had abdominal and pelvic CT scans before treatment were analyzed retrospectively, resulting in the exclusion of 59 patients because they had already undergone treatment outside the study institution, 14 due to a prior history of cancer or synchronous tumor, and 4 who had no histopathologic report defining the tumor location. After evaluating the CT scans of the remaining 267 women, an additional 59 were excluded due to scan quality (eg, hardware artifact or image quality). This cohort study was thus based on the final 208 patients.

Sociodemographic data and clinical information related to their treatment were collected in the electronic and physical case records, including age, ethnic group, reported comorbidities, histologic type and staging of cancer, type of treatment performed, and 1-year survival. Staging was performed based on the International Federation of Gynecology and $\mathrm{Ob}$ stetrics (FIGO) characteristic for EC. ${ }^{17}$

Body composition analysis was performed using CT scans of the patients' abdomen and pelvis available up to 30 days before treatment initiation. Slices located at the third lumbar vertebra (L3) were analyzed by a trained observer with the aid of the sliceOmatic software program 5.0 (TomoVision, Magog, Canada), which enables specific demarcation of skeletal muscle, expressed in HU. Skeletal muscle index $\left(\mathrm{SMI} ; \mathrm{cm}^{2} / \mathrm{m}^{2}\right)$ was quantified using the reference radiation attenuation ranging from -29 to +150 $\mathrm{HU}^{11}$ and, after normalization by the square of the 
stature, represented the overall area of skeletal muscle obtained by the L3 scan.

The overall skeletal muscle range was then divided into 2 subranges: the area of skeletal muscle in the range -29 to $+29 \mathrm{HU}$ was denominated as lowradiodensity SMI (LRSMI; $\mathrm{cm}^{2} / \mathrm{m}^{2}$ ), and the area in the range +30 to $+150 \mathrm{HU}$ was denominated as high-radiodensity SMI (HRSMI), representing the cross-sectional muscle area with low and high attenuation, respectively.

It was thus possible to appraise skeletal muscle tissue according to several different aspects, namely (1) the standard SMI, classically used in the literature for diagnosis of sarcopenia, which comprises the overall skeletal muscle attenuation; (2) the skeletal muscle area with lower attenuation and presumably increased intramyocellular triglycerides (LRSMI); and (3) the skeletal muscle area with higher attenuation (HRSMI).

Furthermore, to compare the additional value of our proposed methodology in classifying skeletal muscle according to low or high attenuation, the radiation attenuation for the overall skeletal muscle was also assessed by calculating the average HU value of the total muscle area within the range -29 to $+150 \mathrm{HU}$, because this is the methodology widely used in previous studies to characterize myosteatosis. ${ }^{7,8,18-20}$ Because there is no established cutoff point for these variables, they were categorized according to the population distribution quartiles (eg, HRSMI $<\mathrm{Q} 1$; $\geq \mathrm{Q} 1$ HRSMI <Q2; $\geq \mathrm{Q} 2$ HRSMI <Q3; HRSMI $\geq Q$ 3; Table 1). The radiation attenuation ranges used for the 3 skeletal muscle parameters are summarized in Figure 1 to facilitate comprehension of our methodology and results.

SMI was categorized as below and above the median of our own population; and the average muscle radiation attenuation (AMA) was categorized as below or above $30 \mathrm{HU}$ to construct 4 skeletal muscle phenotypes: "high SMI + high AMA"; "high SMI + low AMA"; "low SMI + high AMA"; and "low SMI + low AMA".

The adipose tissue was quantified using the reference radiation attenuation ranging from -190 to $-30 \mathrm{HU}$ for subcutaneous and intramuscular adipose tissue and -50 to $-150 \mathrm{HU}$ for the visceral adipose tissue. ${ }^{11}$ The estimated total body fat mass was calculated through regression equations developed by Mourtzakis et al, ${ }^{21}$ expressed in kilograms and nor- malized by the square of the stature to generate the fat mass index (FMI; $\mathrm{kg} / \mathrm{m}^{2}$ ).

Statistical analysis was performed using SPSS version 17.0 (SPSS Inc., Chicago, IL). Histograms were plotted to evaluate the symmetry of the variables' distribution curve. Variables were considered normally distributed. In the description of the sample, the data were expressed in average and standard deviation for continuous variables, and percentage for nominal variables. Associations between the categorical variables were analyzed by the chi-square test.

One-year survival was estimated by the KaplanMeier method, and statistical significance among groups was estimated by the log-rank test. Those who remained alive within 365 days based on the date of first cancer treatment were censored. All independent variables assessed by the log-rank test that showed $P \leq .20$ were considered for entering the Cox univariate regression model: age, histologic type, staging, comorbidities (eg, coexistence of systemic arterial hypertension and diabetes mellitus), type of cancer treatment, FMI, and the skeletal muscle parameters: SMI, HRSMI, LRSMI, and AMA. The Cox multiple regression analysis was performed according to the stepwise forward method, wherein the variables that showed $P \leq .20$ in the Cox univariate analysis were included. First, skeletal muscle parameters were included in the models as continuous variables, which allowed for comparison of the same magnitude because they have the same unit of measurement $\left(\mathrm{cm}^{2} / \mathrm{m}^{2}\right)$.

The Kaplan-Meier method and the Cox multiple regression analysis were also used to test the association between the skeletal muscle phenotypes with 1-year mortality. The magnitude of association was determined by the hazard ratio (HR), and variables that showed $P<.05$ were maintained in the final model. Two-sided $P$ values $\leq .05$ were accepted as statistically significant.

\section{Results}

The study group was made up of 208 eligible women with an average age of $64.2 \pm 9.5$ years. Low SMI was associated with sarcoma histologic type and a coexistent diagnosis of diabetes mellitus and systemic arterial hypertension (Table 2). 
Rodrigues and Chaves

\begin{tabular}{|c|c|}
\hline Skeletal Muscle Parameters ${ }^{\mathrm{a}}$ & Quartiles \\
\hline \multicolumn{2}{|l|}{$\mathrm{SMI}, \mathrm{cm}^{2} / \mathrm{m}^{2}$} \\
\hline 1st quartile & 38.37 \\
\hline 2nd quartile & 42.45 \\
\hline 3rd quartile & 49.37 \\
\hline \multicolumn{2}{|c|}{ Average skeletal muscle attenuation, $\mathrm{HU}$} \\
\hline 1st quartile & 21.82 \\
\hline 2nd quartile & 28.01 \\
\hline 3rd quartile & 34.45 \\
\hline \multicolumn{2}{|l|}{ Low-radiodensity SMI, $\mathrm{cm}^{2} / \mathrm{m}^{2}$} \\
\hline 1st quartile & 16.28 \\
\hline 2nd quartile & 22.21 \\
\hline 3rd quartile & 27.04 \\
\hline \multicolumn{2}{|l|}{ High-radiodensity $\mathrm{SMI}, \mathrm{cm}^{2} / \mathrm{m}^{2}$} \\
\hline 1st quartile & 16.19 \\
\hline 2nd quartile & 20.89 \\
\hline 3rd quartile & 27.08 \\
\hline \multicolumn{2}{|l|}{ Fat mass index, $\mathrm{kg} / \mathrm{m}^{2}$} \\
\hline 1st quartile & 8.95 \\
\hline 2nd quartile & 10.87 \\
\hline 3rd quartile & 13.88 \\
\hline
\end{tabular}

Abbreviations: $\mathrm{HU}$, Hounsfield units; SMI, skeletal muscle index. aSMI was calculated in the range -29 to $+150 \mathrm{HU}$, representing the overall skeletal muscle. The subranges low- and high-radiodensity SMI were calculated using the skeletal muscle area in range -29 to $+29 \mathrm{HU}$ and +30 to $+150 \mathrm{HU}$, respectively.

The highest median survival was observed among patients with the high SMI + high AMA and low SMI + high AMA skeletal muscle phenotypes, whereas shorter median survival was found in those with the low SMI + low AMA phenotype. Interestingly, there was no statistical difference in median survival between the high SMI + high AMA and low SMI + high AMA phenotypes (Table 3, Figure 2).
Cox multiple regression analysis, after adjusting for confounding factors, showed that both AMA and HRSMI presented the best protective factor for 1-year survival (patients were 11\% less likely to die with each increment in $1 \mathrm{~cm}^{2} / \mathrm{m}^{2}$ ), followed by LRSMI. SMI as a continuous variable did not predict 1 -year survival (Table 4). When the skeletal muscle phenotypes were considered in the Cox multiple regression analysis, only low SMI + low AMA showed an association with 1-year mortality. FMI was a protective factor for this outcome. Similar results were obtained when the regression was performed with or without the sarcoma histologic type included (Table 5).

\section{Discussion}

In the present study, all the skeletal muscle parameters evaluated proved to be related to adverse prognosis, being significantly associated with 1 -year survival, except for the SMI. Considering the different analysis appraised, AMA and HRSMI showed the best association with 1-year mortality.

Low SMI was found in $26.4 \%$ of the patients and was significantly more frequent among those with the sarcoma histologic type. Metabolic and inflammatory alterations that occur more markedly in aggressive cancers result in an increase in proinflammatory cytokine levels, hyperinsulinemia, and a reduction in calorie intake. These factors may be a plausible explanation for the worsening in nutritional status, because they promote loss of body mass, including muscle mass and adipose tissue. ${ }^{22} \mathrm{~A}$ direct association between skeletal muscle attenuation and the host systemic inflammatory response in patients with operable primary colorectal cancer has been also described..$^{23}$ After appraising the

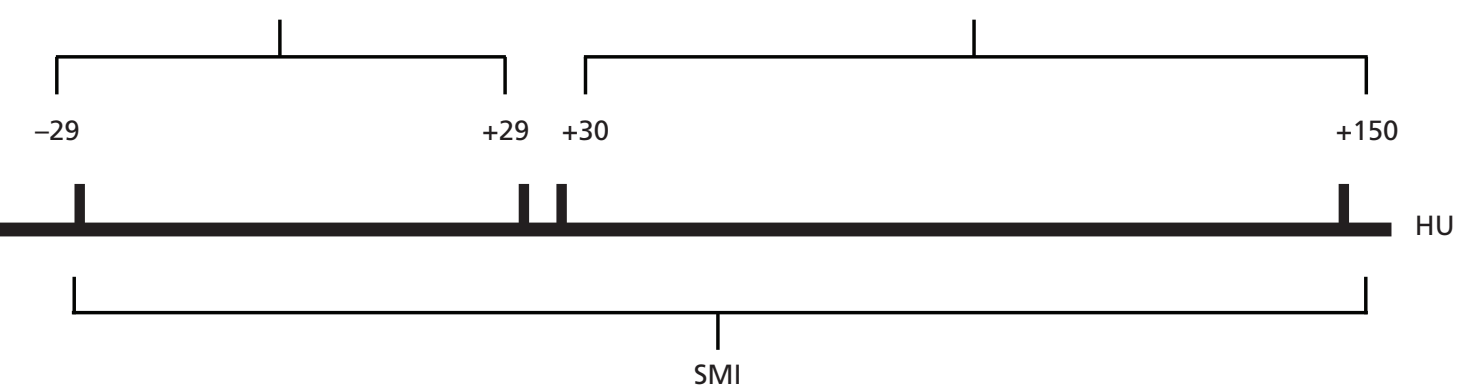

Figure 1. Skeletal muscle classification purpose according to subranges of radiodensity. Abbreviations: HU, Hounsfield units; SMI, skeletal muscle index. 
Muscle Quality and Short-Term Survival

\begin{tabular}{|c|c|c|c|c|}
\hline Variables & $\begin{array}{l}\text { Total } \\
\text { n (\%) }\end{array}$ & $\begin{array}{c}\text { Normal SMI } \\
\text { n (\%) }\end{array}$ & $\begin{array}{c}\text { Low SMI } \\
\text { n (\%) }\end{array}$ & $P$ Value $^{\mathrm{a}}$ \\
\hline Age, y & & & & .224 \\
\hline$<65$ & $102(49.1)$ & $56(53.8)$ & $45(43.3)$ & \\
\hline$\geq 65$ & $106(50.9)$ & $48(46.2)$ & $59(56.7)$ & \\
\hline Race/Ethnicity & & & & .954 \\
\hline Caucasian & $116(56.0)$ & $58(55.8)$ & $58(56.3)$ & \\
\hline Mixed (brown) & $64(30.9)$ & $33(31.7)$ & $31(30.1)$ & \\
\hline Black & $27(13.0)$ & $13(12.5)$ & $14(13.6)$ & \\
\hline \multicolumn{5}{|l|}{ Histopathologic characteristics } \\
\hline Histologic type & & & & .051 \\
\hline Adenocarcinoma & $177(85.1)$ & $93(89.4)$ & $84(80.7)$ & \\
\hline Sarcoma & $31(14.9)$ & $11(10.6)$ & $20(19.2)$ & \\
\hline Histologic subtype & & & & .146 \\
\hline Endometrioid & $96(52.7)$ & $56(57.1)$ & $40(48.2)$ & \\
\hline Nonendometrioid $^{\mathrm{b}}$ & $86(47.3)$ & $42(42.9)$ & $43(51.8)$ & \\
\hline Cancer stage & & & & .146 \\
\hline Stage $I / I I$ & $102(52.3)$ & $57(56.5)$ & $45(47.9)$ & \\
\hline Stage III/IV & $93(47.3)$ & $44(43.6)$ & $59(52.1)$ & \\
\hline Comorbidities & & & & .005 \\
\hline SAH & $90(57.4)$ & $38(46.9)$ & $52(68.4)$ & \\
\hline $\mathrm{DM}$ & $4(2.5)$ & $2(2.5)$ & $2(2.6)$ & \\
\hline $\mathrm{SAH}+\mathrm{DM}$ & $59(37.6)$ & $41(50.6)$ & $18(23.7)$ & \\
\hline Others ${ }^{c}$ & $4(2.5)$ & $0(0)$ & $4(5.3)$ & \\
\hline Type of cancer treatment & & & & .065 \\
\hline Surgery & $111(53.3)$ & $55(49.6)$ & $56(57.8)$ & \\
\hline Surgery + adjuvant chemotherapy & $66(31.7)$ & $41(36.9)$ & $25(25.7)$ & \\
\hline Palliative treatment & $31(14.9)$ & $15(13.5)$ & $16(16.5)$ & \\
\hline
\end{tabular}

Abbreviations: DM, diabetes mellitus; SAH, systemic arterial hypertension; SMI, skeletal muscle index.

aChi-square test.

bSerous, mucinous, and clear cells.

chronic obstructive pulmonary disease and cardiovascular disease.

nutritional status of women with gynecologic cancer through the Patient-Generated Subjective Global Assessment, Rodrigues and Chaves ${ }^{24}$ identified a significant association between the advanced stage of cancer and the greater frequency of nutritional risk or severe malnutrition.

Low SMI is a well-consolidated predictor of mortality in oncology. A recent meta-analysis assessing the impact of sarcopenia on the mortality of patients with solid tumor cancer concluded that sarcopenia is associated with the worsening of overall survival, irrespective of the type and stage of cancer. ${ }^{5}$ Although other studies have previously demonstrated decreased survival in patients with cancer with low muscle attenuation, which is in accordance with the present study, they used a different approach to define muscle quality, which was limited to determine the average muscle attenuation for the comparison of clinical and surgical outcomes. ${ }^{8,18-20,23}$ We hypothesize that the determination of the low/high radiodensity area is more appropriate in characterizing muscle quality than merely classifying the individual according to their average muscle attenuation, because it allows identification of the amount of skeletal muscle area with high- or low-radiodensity. To our knowledge, no studies have been reported that use a similar methodology to investigate muscle radiation attenuation. Our results suggest that a higher amount of high-radiodensity muscle has a similar protective 
Rodrigues and Chaves

\begin{tabular}{|c|c|c|c|c|}
\hline \multirow[b]{2}{*}{ Skeletal Muscle Parameters } & \multicolumn{4}{|c|}{ 1-Year Survival } \\
\hline & Events (\%) & Median & $95 \% \mathrm{Cl}$ & Log-Rank $P$ Value \\
\hline \multicolumn{5}{|l|}{$\mathrm{SMI}^{\mathrm{a}}$} \\
\hline $\operatorname{High}\left(\geq 42.4 \mathrm{~cm}^{2} / \mathrm{m}^{2}\right)$ & $20(28.9)$ & 329.42 & $312.1-346.6$ & \multirow{2}{*}{.01} \\
\hline Low $\left(<42.4 \mathrm{~cm}^{2} / \mathrm{m}^{2}\right)$ & $49(71.0)$ & 233.74 & $204.4-263.0$ & \\
\hline \multicolumn{5}{|c|}{ Average muscle attenuation, $\mathrm{HU}$} \\
\hline High AMA ( $\geq 30 \mathrm{HU})$ & $10(14.5)$ & 342.4 & $325.74-359.2$ & \multirow{2}{*}{.01} \\
\hline Low AMA (<30 HU) & $59(85.5)$ & 241.9 & $216.2-267.7$ & \\
\hline \multicolumn{5}{|l|}{ Skeletal muscle phenotypes ${ }^{b}$} \\
\hline High SMI + high AMA & $4(5.7)$ & $355.30^{c}$ & $341.1-369.4$ & \multirow{4}{*}{.01} \\
\hline Low SMI + high AMA & $6(8.7)$ & $323.42^{c, d}$ & $288.5-358.2$ & \\
\hline High SMI + low AMA & $16(23.2)$ & $306.36^{d}$ & $277.6-335.0$ & \\
\hline Low SMI + low AMA & $43(62.4)$ & $192.05^{\mathrm{e}}$ & $156.1-227.9$ & \\
\hline
\end{tabular}

Abbreviations: AMA, average muscle radiation attenuation; HU, Hounsfield units; SMI, skeletal muscle index.

aSMI range: -29 to $+150 \mathrm{HU}$.

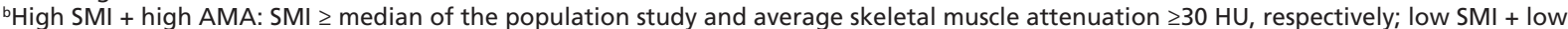

AMA: $\mathrm{SMI}<$ median of the population study and average skeletal muscle attenuation $<30 \mathrm{HU}$, respectively.

'No statistical difference was seen between high SMI + high AMA and low SMI + high AMA.

dNo statistical difference was seen low SMI + high AMA and high SMI + low AMA.

eStatistical difference was seen between low SMI + low AMA and all other phenotypes.

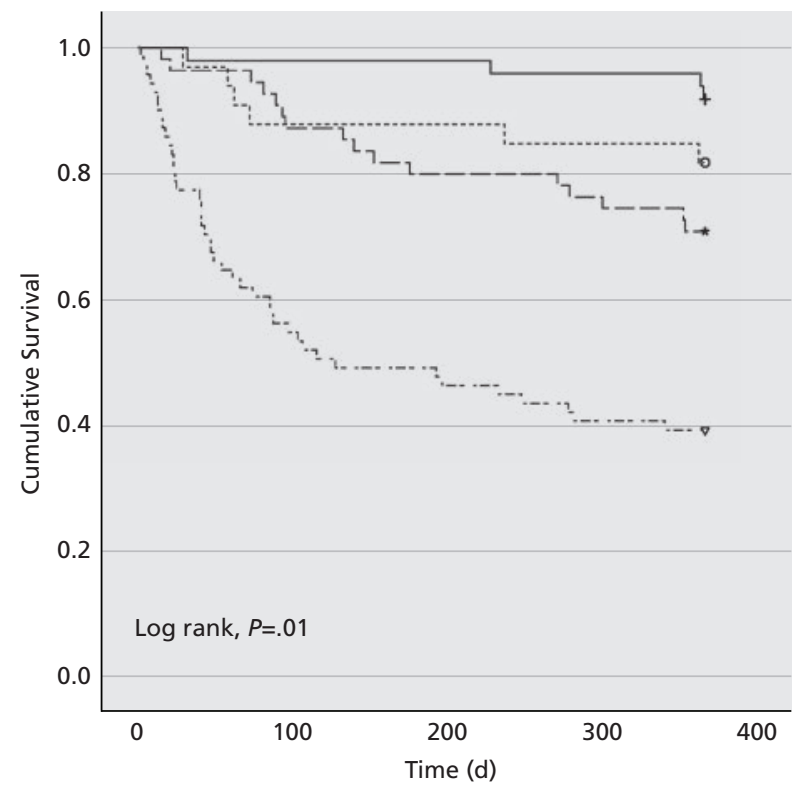

Skeletal Muscle Phenotypes

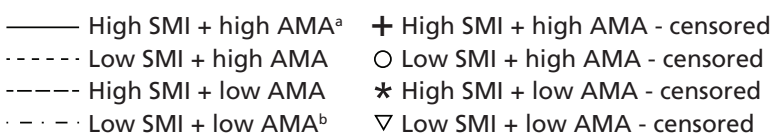

Figure 2. Kaplan-Meier curve for 1-year survival according to the skeletal muscle phenotypes proposed.

Abbreviations: AMA, average muscle radiation attenuation; HU, Hounsfield units; SMI, skeletal muscle index (calculated in range -29 to $+150 \mathrm{HU}$ ).

aHigh SMI + high AMA: SMI $\geq$ median of the population study and average skeletal muscle attenuation $\geq 30 \mathrm{HU}$, respectively.

'Low SMI + low AMA: SMI < median of the population study and average skeletal muscle attenuation $<30 \mathrm{HU}$, respectively. effect on short-term survival compared with high AMA.

Because our results show that skeletal muscle characteristics, related to either quantity or quality, may coexist, evaluating cancer prognosis according to skeletal muscle phenotype is a promising tool. Considering that we did not find a statistical difference in median survival time between the phenotypes groups high SMI + high AMA versus low SMI + high AMA, we hypothesize that there is a minimum amount of high-radiodensity muscle that exerts a protective effect in cancer prognosis, despite the presence of low SMI. This may also be the explanation for the lack of association between SMI and mortality.

Thus, the additional value of using HRSMI for the construction of skeletal muscle phenotypes to assess cancer prognosis should be exploited in the future. Further studies should explore this finding in a greater sample size, with different cancer outcomes, and consider the evaluation of other phenotypes than the proposed phenotypes in our study, especially with regard to the inclusion of fat mass. It is also important to consider the assessment of functional capacity in patients with cancer with different skeletal muscle phenotypes. Interestingly, muscle quality (assessed based on 
Muscle Quality and Short-Term Survival

Table 4. Cox Multiple Regression Analysis for 1-Year Survival Based on Different Skeletal Muscle Parameters

\begin{tabular}{|c|c|c|c|c|}
\hline & \multicolumn{2}{|c|}{ Unadjusted } & \multicolumn{2}{|c|}{ Adjusted $^{a}$} \\
\hline & HR $(95 \% \mathrm{Cl})$ & $P$ Value & HR $(95 \% \mathrm{Cl})$ & $P$ Value \\
\hline Model 1: SMI $\left(\mathrm{cm}^{2} / \mathrm{m}^{2}\right)$ & $0.92(0.89-0.95)$ & .001 & $0.96(0.91-1.07)$ & .096 \\
\hline Model 2: HRSMI $\left(\mathrm{cm}^{2} / \mathrm{m}^{2}\right)$ & $0.89(0.86-0.93)$ & .001 & $0.89(0.84-0.93)$ & .001 \\
\hline Model 3: LRSMI $\left(\mathrm{cm}^{2} / \mathrm{m}^{2}\right)$ & $1.03(0.99-1.06)$ & .055 & $1.08(1.02-1.14)$ & .003 \\
\hline Model 4: Average muscle attenuation $(\mathrm{HU})$ & $0.92(0.89-0.94)$ & .001 & $0.89(0.85-0.94)$ & .001 \\
\hline
\end{tabular}

Abbreviations: HR, hazard ratio; HRSMI, high-radiodensity skeletal muscle index (skeletal muscle in the range +30 to $+150 \mathrm{HU}$ ); HU, Hounsfield units; LRSMI, low-radiodensity skeletal muscle index (skeletal muscle in the range -29 to $+29 \mathrm{HU}$ ); SMI, skeletal muscle index.

${ }^{a}$ Adjusted model for age, histologic type, staging, comorbidities (eg, systemic arterial hypertension and diabetes mellitus), type of cancer treatment, and fat mass index $\left(\mathrm{kg} / \mathrm{m}^{2}\right)$.

muscle-strength), regardless of the quantity (determined by dual-energy $\mathrm{x}$-ray absorptiometry), has already been shown to be determinant for decreasing the risk of aggravation of functional capacity in healthy elderly individuals. ${ }^{25}$

Lastly, FMI likewise proved to be a protective factor for early mortality. Studies evaluating the role of FMI in cancer outcomes are scarce. However, this obesity paradox has already been shown for other chronic diseases and certain types of cancer. ${ }^{26-28}$

The principal limitation of the study is its retrospective design, which restricts the gathering of data in relation to the quality of information obtained and due to the amount of absent data. Moreover,

\section{Table 5. Cox Regression Analysis for 1-Year Survival Based on Different Skeletal Muscle Phenotypes}

\begin{tabular}{|c|c|c|c|c|c|c|c|c|}
\hline \multirow{3}{*}{$\begin{array}{l}\text { Variables Included } \\
\text { in the Final Model }\end{array}$} & \multicolumn{4}{|c|}{ Overall Population $(\mathrm{N}=208)$} & \multicolumn{4}{|c|}{ Adenocarcinoma $(\mathrm{N}=177)$} \\
\hline & \multicolumn{2}{|c|}{ Unadjusted } & \multicolumn{2}{|c|}{ Adjusted } & \multicolumn{2}{|c|}{ Unadjusted } & \multicolumn{2}{|c|}{ Adjusted } \\
\hline & HR $(95 \% \mathrm{Cl})$ & $P$ Value & HR $(95 \% \mathrm{Cl})$ & $P$ Value & HR $(95 \% \mathrm{Cl})$ & $P$ Value & $\mathrm{HR}(95 \% \mathrm{Cl})$ & $P$ Value \\
\hline \multicolumn{9}{|c|}{ Skeletal muscle phenotypes ${ }^{a}$} \\
\hline High SMI + high AMA & 1.00 (Ref) & - & 1.00 (Ref) & - & 1.00 (Ref) & - & 1.00 (Ref) & - \\
\hline Low SMI + high AMA & $2.39(0.67-8.47)$ & .716 & $2.10(0.48-9.16)$ & .332 & $2.06(0.55-7.70)$ & .279 & $2.15(0.49-9.42)$ & .308 \\
\hline High SMI + low AMA & $3.93(1.31-11.78)$ & .014 & $2.20(0.67-7.16)$ & .191 & $2.62(0.82-8.36)$ & .103 & $1.79(0.49-6.48)$ & .370 \\
\hline Low SMI + low AMA & $11.89(4.26-33.19)$ & .001 & $5.31(1.71-16.51)$ & .004 & $9.95(3.50-28.26)$ & .001 & $5.09(1.56-16.61)$ & .007 \\
\hline Fat mass index, $\mathrm{kg} / \mathrm{m}^{2}$ & $0.88(0.81-0.95)$ & .002 & $0.86(0.77-0.96)$ & .012 & $0.85(0.78-0.94)$ & .002 & $0.84(0.73-0.97)$ & .018 \\
\hline Age, y & $1.02(0.99-1.04)$ & .061 & $0.99(0.96-1.02)$ & .479 & $1.03(1.00-1.05)$ & .042 & $0.99(0.95-1.03)$ & .858 \\
\hline \multicolumn{9}{|l|}{ Histologic type } \\
\hline Adenocarcinoma & 1.00 (Ref) & - & 1.00 (Ref) & - & - & - & - & - \\
\hline Sarcoma & $2.71(1.59-4.61)$ & .001 & $2.66(1.35-5.26)$ & .005 & - & - & - & - \\
\hline \multicolumn{9}{|l|}{ Staging } \\
\hline Stages I/II & 1.00 (Ref) & - & 1.00 (Ref) & - & 1.00 (Ref) & - & 1.00 (Ref) & - \\
\hline Stages III/IV & $4.39(2.36-8.17)$ & .001 & $3.03(1.41-6.52)$ & .005 & $3.58(1.82-7.03)$ & .001 & $1.78(0.69-4.58)$ & .229 \\
\hline \multicolumn{9}{|l|}{ Type of cancer treatment } \\
\hline Curative surgery & 1.00 (Ref) & - & 1.00 (Ref) & - & 1.00 (Ref) & - & 1.00 (Ref) & - \\
\hline $\begin{array}{l}\text { Surgery + adjuvant } \\
\text { chemotherapy }\end{array}$ & $0.68(0.34-1.37)$ & .287 & $0.40(0.16-1.00)$ & .051 & $0.88(0.38-2.04)$ & .778 & $0.90(0.28-2.86)$ & .909 \\
\hline $\begin{array}{l}\text { Palliative } \\
\text { chemotherapy }\end{array}$ & $5.57(3.29-9.43)$ & .001 & $1.06(0.49-2.28)$ & .866 & $9.59(5.09-18.07)$ & .001 & $1.70(0.62-4.66)$ & .301 \\
\hline \multicolumn{9}{|l|}{ Comorbidities (SAH + DM) } \\
\hline No & 1.00 (Ref) & - & 1.00 (Ref) & - & 1.00 (Ref) & - & 1.00 (Ref) & - \\
\hline Yes & $1.54(0.92-2.59)$ & .097 & $2.66(1.33-5.31)$ & .005 & $1.69(0.91-3.12)$ & .092 & $2.31(1.07-4.96)$ & .032 \\
\hline
\end{tabular}

Abbreviations: AMA, average muscle radiation attenuation; DM, diabetes mellitus; HR, hazard ratio; HU, Hounsfield unites; SAH, systemic arterial hypertension; SMI, skeletal muscle index (calculated in range -29 to $+150 \mathrm{HU}$ ).

a High SMI + high AMA: SMI $\geq$ median of the population study and average skeletal muscle attenuation $\geq 30 \mathrm{HU}$, respectively; low SMI + low AMA:

$\mathrm{SMI}<$ median of the population study and average skeletal muscle attenuation <30 HU, respectively. 
because the cohort consisted only of women who had CT examinations before treatment, and given that this examination is not routinely performed in all cases, our sample comprised patients with a greater incidence of advanced-stage cancer, sarcoma-type histology, and nonendometrioid histologic subtype, which is different from what is expected in the ordinary EC population and interferes in the prognosis of this tumor. ${ }^{29}$

However, although the mortality rate was higher than expected for EC, after adjusting the result to consider these potentially confounding variables for 1 -year survival, all of the skeletal muscle parameters persisted as independent predictors of survival in the Cox regression model.

\section{Conclusions}

We found that classifying skeletal muscle into subranges of radiodensity had an additional value compared with using the overall skeletal muscle area (SMI). These results reinforce the need to expand investigations in this area, because a combination of unfavorable phenotypes of body composition can worsen the impact on patient outcomes. Particular attention must be given to the low SMI + low AMA phenotype. Even so, there remained the need to define a cutoff point for classifying skeletal muscle quality, both with respect to HRSMI and LRSMI. The additional value of using HRSMI and LRSMI in skeletal muscle phenotypes to assess cancer survival and other outcomes should be exploited in future studies.

\section{References}

1. Cruz-Jentoft AJ, Baeyens JP, Bauer JM, et al. Sarcopenia: European consensus on definition and diagnosis: report of the European Working Group on Sarcopenia in Older People. Age Ageing 2010;39:412-423.

2. Thibault R, Genton L, Pichard C. Body composition: why, when and for who? Clin Nutr 2012;31:435-447.

3. Palmela C, Velho S, Agostinho L, et al. Body composition as a prognostic factor of neoadjuvant chemotherapy toxicity and outcome in patients with locally advanced gastric cancer. J Gastric Cancer 2017;17:74.

4. van Vugt JL, Levolger S, Coelen RJ, et al. The impact of sarcopenia on survival and complications in surgical oncology: a review of the current literature. J Surg Oncol 2015;112:681-682.

5. Shachar SS, Williams GR, Muss HB, Nishijima TF. Prognostic value of sarcopenia in adults with solid tumours: a meta-analysis and systematic review. Eur J Cancer 2016;57:58-67.

6. Aubrey J, Esfandiari N, Baracos VE, et al. Measurement of skeletal muscle radiation attenuation and basis of its biological variation. Acta Physiol (Oxf) 2014;210:489-497.

7. Rollins KE, Tewari N, Ackner A, et al. The impact of sarcopenia and myosteatosis on outcomes of unresectable pancreatic cancer or distal cholangiocarcinoma. Clin Nutr 2016;35:1103-1109.

8. Kaibori $\mathrm{M}$, Ishizaki $\mathrm{M}$, Iida $\mathrm{H}$, et al. Effect of intramuscular adipose tissue content on prognosis in patients undergoing hepatocellular carcinoma resection. J Gastrointest Surg 2015;19:1315-1323.

9. Dériaz $\mathrm{O}$, Dumont $\mathrm{M}$, Bergeron $\mathrm{N}$, et al. Skeletal muscle low attenuation area and maximal fat oxidation rate during submaximal exercise in male obese individuals. Int J Obes Relat Metab Disord 2001;25:1579-1584.

10. Goodpaster BH, Thaete FL, Kelley DE. Thigh adipose tissue distribution is associated with insulin resistance in obesity and in type 2 diabetes mellitus. Am J Clin Nutr 2000;71:885-892

11. Mitsiopoulos N, Baumgartner RN, Heymsfield SB, et al. Cadaver validation of skeletal muscle measurement by magnetic resonance imaging and computerized tomography. J Appl Physiol (1985) 1998;85:115-122.

12. Kim YJ, Park JW, Kim JW, et al. Computerized automated quantification of subcutaneous and visceral adipose tissue from computed tomography scans: development and validation study. JMIR Med Inform 2016;4:e2.

13. Lee S. Exercise without weight loss is an effective strategy for obesity reduction in obese individuals with and without type 2 diabetes. J Appl Physiol (1985) 2005;99:1220-1225.

14. Goodpaster BH, Carlson CL, Visser M, et al. Attenuation of skeletal muscle and strength in the elderly: the Health ABC Study. J Appl Physiol (1985) 2001;90:2157-2165.

15. Taaffe DR, Henwood TR, Nalls MA, et al. Alterations in muscle attenuation following detraining and retraining in resistance-trained older adults. Gerontology 2008;55:217-223

16. Visser M, Goodpaster BH, Kritchevsky SB, et al. Muscle mass, muscle strength, and muscle fat infiltration as predictors of incident mobility limitations in well-functioning older persons. J Gerontol A Biol Sci Med Sci 2005;60:324-333.

17. Pecorelli $S$. Revised FIGO staging for carcinoma of the vulva, cervix, and endometrium. Int J Gynaecol Obstet 2009;105:103-104.

18. Fujiwara N, Nakagawa $\mathrm{H}$, Kudo $\mathrm{Y}$, et al. Sarcopenia, intramuscular fat deposition, and visceral adiposity independently predict the outcomes of hepatocellular carcinoma. J Hepatol 2015;63:131-140

19. van Dijk DP, Bakens MJ, Coolsen MM, et al. Low skeletal muscle radiation attenuation and visceral adiposity are associated with overall survival and surgical site infections in patients with pancreatic cancer. J Cachexia Sarcopenia Muscle 2017;8:317-326.

20. Okumura $S$, Kaido T, Hamaguchi $Y$, et al. Impact of preoperative quality as well as quantity of skeletal muscle on survival after resection of pancreatic cancer. Surgery 2015;157:1088-1098.

21. Mourtzakis M, Prado CM, Lieffers JR, et al. A practical and precise approach to quantification of body composition in cancer patients using computed tomography images acquired during routine care. Appl Physiol Nutr Metab 2008;33:997-1006.

22. Fearon $\mathrm{K}$, Arends J, Baracos V. Understanding the mechanisms and treatment options in cancer cachexia. Nat Rev Clin Oncol 2012;10:90-99.

23. Malietzis $\mathrm{G}$, Johns $\mathrm{N}, \mathrm{Al}$-Hassi $\mathrm{HO}$, et al. Low muscularity and myosteatosis is related to the host systemic inflammatory response in patients undergoing surgery for colorectal cancer. Ann Surg 2016;263:320-325

24. Rodrigues CS, Chaves GV. Patient-generated subjective global assessment in relation to site, stage of the illness, reason for hospital admission, and mortality in patients with gynecological tumors. Support Care Cancer 2015;23:871-879.

25. Barbat-Artigas S, Rolland Y, Vellas B, Aubertin-Leheudre M. Muscle quantity is not synonymous with muscle quality. J Am Med Dir Assoc 2013;14:852.e1-7.

26. Flegal KM, Kit BK, Orpana H, Graubard BI. Association of all-cause mortality with overweight and obesity using standard body mass index categories: a systematic review and meta-analysis. JAMA 2013;309:71-82.

27. Calle EE, Rodriguez C, Walker-Thurmond K, Thun MJ. Overweight, obesity, and mortality from cancer in a prospectively studied cohort of U.S. adults. N Engl J Med 2003;348:1625-1638.

28. Zhang SS, Yang H, Luo KJ, et al. The impact of body mass index on complication and survival in resected oesophageal cancer: a clinical-based cohort and meta-analysis. Br J Cancer 2013;109:2894-2903.

29. Madison T, Schottenfeld D, James SA, et al. Endometrial cancer socioeconomic status and racial/ethnic differences in stage at diagnosis, treatment, and survival. Am J Public Health 2004;94:2104-2111. 